\title{
Erratum to: Guidance for the treatment of deep vein thrombosis and pulmonary embolism
}

\author{
Michael B. Streiff ${ }^{1} \cdot$ Giancarlo Agnelli $^{2} \cdot$ Jean M. Connors $^{3} \cdot$ Mark Crowther $^{4}$.

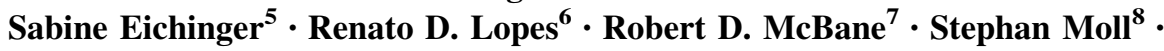 \\ Jack Ansell ${ }^{9}$
}

Published online: 5 March 2016

(C) Springer Science+Business Media New York 2016

\section{Erratum to: J Thromb Thrombolysis (2016) \\ 41:32-67 \\ DOI 10.1007/s11239-015-1317-0}

In Table 12, the dose reduction recommendation for apixaban is incorrect. The recommendation "In patients with at least 2 of the following characteristics: age $\geq 80$ years, body weight $\leq 60 \mathrm{~kg}$, or serum creatinine $\geq 1.5 \mathrm{mg} / \mathrm{dL}$, the recommended dose is $2.5 \mathrm{mg}$ orally BID" only applies to patients with atrial fibrillation not VTE patients. Also, the middle name for the co-author Renato D. Lopes was not provided in the accepted version and published. It is corrected with this erratum.

The online version of the original article can be found under doi:10.1007/s11239-015-1317-0.

Michael B. Streiff

mstreif@jhmi.edu

1 Division of Hematology, Department of Medicine and Pathology, The Johns Hopkins University School of Medicine, Baltimore, MD, USA

2 Stroke Unit, Department of Internal Medicine, University of Perugia, Perugia, Italy

3 Hematology Division, Brigham and Women's Hospital, Dana Farber Cancer Institute, Harvard Medical School, Boston, MA, USA

4 Departments of Medicine and Pathology and Molecular Medicine, McMaster University, Hamilton, Canada
5 Department of Medicine, Medical University of Vienna, Vienna, Austria

6 Division of Cardiology, Department of Medicine, Duke University Medical Center, Durham, NC, USA

7 Cardiovascular Division, Department of Medicine, Mayo Clinic, Rochester, MN, USA

8 Department of Medicine, University of North Carolina School of Medicine, Chapel Hill, NC, USA

9 Department of Medicine, Hofstra North Shore/LIJ School of Medicine, Hempstead, NY, USA 